Case report

\title{
Dupuytren's contracture treated with botulinum toxin A injection
}

\author{
Shane Michael Davis*,1, Eric Yao Chang² \\ ${ }^{1}$ University of California, Irvine, CA, USA; ${ }^{2}$ Restore Orthopedics \& Spine Center, Orange, CA, USA
}

\begin{abstract}
Dupuytren's contracture is a condition in which fibromatosis of the palmar and digital fascia can lead to painful contractures of the hand. Common conservative treatments are of limited efficacy, while invasive options have risk of adverse effects. A 57-year-old female presented with chronic, bilateral hand pain secondary to Dupuytren's contractures. Traditional conservative treatments did not provide relief. She received a series of three botulinum toxin A injections into the palmar fascia of both hands, resulting in four months of improved hand function and pain relief. This case presents a novel treatment for Dupuytren's contracture using botulinum toxin injection. Botulinum toxin has inhibitory effects on the neuromuscular junction and pain signaling pathways to relax muscles and reduce pain. We believe its action on this patient's intrinsic hand muscles resulted in her improved hand function. Botulinum toxin injection can be considered as a treatment option for Dupuytren's contracture.
\end{abstract}

Keywords: Dupuytren contracture; botulinum toxin; hand; pain; injections

\section{Introduction}

Dupuytren's contracture is a disease state characterized by progressive hyperplasia of the palmar fascia. It has a slow onset, typically beginning as a painless nodule in the palm of the hand, which can progress to painful and debilitating flexion contractures of the metacarpophalangeal and proximal interphalangeal joints, most commonly involving the fourth and fifth digits. The exact cause of the disease is unknown. Histologic studies have shown increased levels of fibroblasts and type III collagen, with myofibroblasts in particular thought to drive abnormal collagen deposition [1].

There is also believed to be an autoimmune component and newer research has suggested that dysfunctional stem cell

Received: December 2020; Accepted after review: March 2021; Published: March 2021.

${ }^{*}$ Corresponding author: Shane Michael Davis, University of California, Irvine, CA 92697, USA

Email: shanedavismd@gmail.com pathways may play a role [2]. While newer treatment options are being investigated to target these pathways, the primary methods continue to be non-invasive measures such as heat, stretching and ultrasound for mild disease, injection of corticosteroid or collagenase as disease progresses, and surgical release through fasciotomy or fasciectomy in refractory or severe cases. All of these modalities have drawbacks and none are without relatively high rates of recurrence [3].

We present a case in which Botulinum toxin $A$ injection was used to successfully treat Dupuytren's contracture in a 57-year-old female who had failed common non-surgical treatments. Botulinum toxin is a common medication used in the management of spasticity, and this is the first reported case of its use in treating myofascial contracture of the palmar fascia. This presents a new, minimallyinvasive treatment option for the management of Dupuytren's contracture. 


\section{Case report}

A 57-year-old female presented to the orthopedic clinic with chronic, bilateral hand pain secondary to Dupuytren's myofascial contractures. She had onset of pain at the triangular area of her left palm three years prior, with similar symptoms presenting in her right palm two years later. At the time of evaluation, she reported that her pain was constant, dull and worse on the left than the right. It was rated $4 / 10$ on average and $9 / 10$ at its worst using a numerical rating scale. The symptoms were exacerbated by pinching or grasping objects in her hands and relieved with rest and pain medication. She reported associated weakness and functional limitations in gripping and fine motor dexterity of her hands but denied numbness, tingling, triggering or pain in her neck, shoulders, elbows or wrists. She had a history of bilateral hand tendonitis and was currently unable to work as a dentist due to her symptoms.

Previous evaluations included that with a rheumatologist, who ruled out rheumatoid arthritis, and a hand surgeon, who administered cortisone injections with minimal, temporary relief. Other treatments she had tried included anti-inflammatory medications (ibuprofen and meloxicam), oral steroids, opiates (tramadol and hydrocodoneacetaminophen), physical therapy and acupuncture. She had not tried splinting, bracing or neuropathic medications such as gabapentin.

Pertinent physical exam findings included: palpable palmar nodules and cords without evidence of contracture (Tubiana Stage N) [4], tenderness over the palm with deep compression bilaterally and limited active range of motion of her intrinsic hand muscles due to pain. Upper extremity strength and sensory examination was otherwise normal. Relevant imaging studies included bilateral hand and wrist MRIs which showed interosseous ganglion cysts bilaterally.

The patient returned to the clinic for injections of 50 units botulinum toxin $A$ (incobotulinumtoxin $\mathrm{A}$, diluted in $4 \mathrm{~mL}$ of normal saline to 1.25 units per $\mathrm{mL}$ ) into the palmar fascia of her left hand, followed by a 25 unit ( 0.625 units per $\mathrm{mL}$ ) injection into her right hand one month later. Injections were placed just above the flexor digitorum tendons at 20 points along the palm (five points per digit, excluding the thumb) (Figure 1).

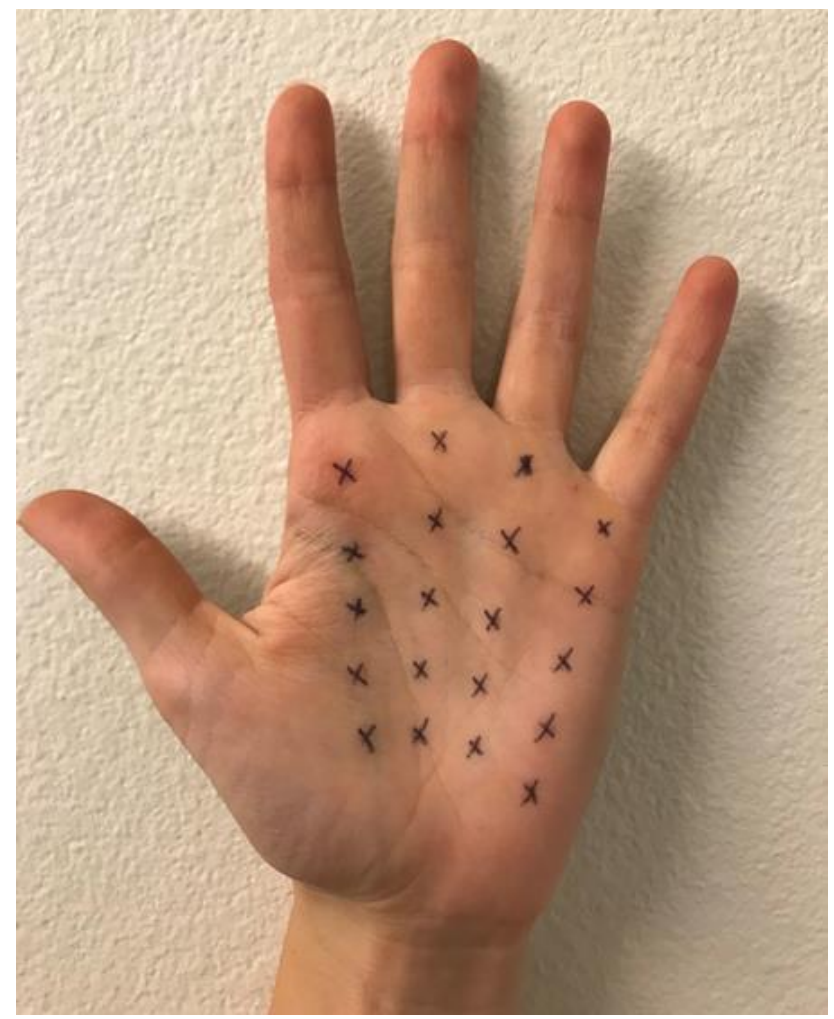

Fig. 1. Palmar injection site locations (representation only, not a patient photo) 
These provided temporary, mild relief. Four months later she returned for 50 unit injections into her bilateral hands using the same technique, providing two to three months of relief. Four months later, she was injected with 100 units (2.5 units per $\mathrm{mL}$ ) into each hand, resulting in three to fourth months of marked improvement in pain (1/10 on average, up to $2 / 10$ at its worst), range of motion and function of her hands. Her only remaining limitation was in fine motor dexterity, but she was successfully able to return to work.

\section{Discussions}

Dupuytren's contracture is a common, debilitating and sometimes painful condition which often recurs despite current treatments. For mild disease, initial interventions usually consist of conservative measures such as gentle exercise, massage or splinting; however, there is no current data showing these measures prevent disease progression. There is also a theoretical concern that stretching of the fascia can actually worsen contractures through tension-related mechanisms which upregulate the activity of fibroblasts and myoblasts, although this has not been proven in clinical studies [5]. Glucocorticoid injections are a commonly used modality for mild-to-moderate disease, especially those with rapid progression and tenderness, signifying a concurrent inflammatory tenosynovitis. A study by Ketchum \& Donahue showed softening or flattening of nodules in $97 \%$ of cases by administration of an average of 3.2 triamcinolone acetonide injections per nodule. While this is a high success rate, about half of the patients experienced recurrence within the next three years and complications included muscle atrophy and rupture of the flexor tendon [6]. One case report on topical corticosteroid use reported good results, while another on oral administration reported no benefit; however, no large studies exist and neither are routinely used in practice [7]. For cases where flexion contracture has developed, traditional treatment options include open fasciectomy, or fasciotomy through open, percutaneous or needle techniques. While open fasciectomy has been considered the most definitive approach, it comes with the highest risk of complications, including scarring, skin tears, infection, pulley rupture and injury to the neurovasculature. There is also debate as to which patients would most benefit and when in the disease process to intervene [8]. Needle fasciotomy is a much less invasive procedure in which the tip of a needle is used to break apart the contracted tissue. There are fewer complications and it can be performed in the office with local anesthetic; however, recurrence rates tend to be high [8]. In recent years, injection of collagenase clostridium histolyticum has also become a preferred intervention as it is a less invasive alternative to surgery with similar efficacy and recurrence rates [9]. Radiotherapy has proven to be somewhat effective through its proposed mechanism to reduce myofibroblast development; however, there are theoretical concerns about radiation exposure inducing cancer in long-term use [7]. Other attempts at using continuous slow skeletal traction, physical therapy, dimethyl sulfoxide, vitamin E, allopurinol and interferon have largely proven to be unsuccessful [10].

While many treatment options exist, there is not a clear consensus of an optimal management algorithm. As a result, management often takes a multi-modal approach, where therapies are escalated as the disease progresses. Additional effective treatment methods are needed, and this case represents the first described use of Botulinum toxin injection for the successful treatment of Dupuytren's contracture in a patient with refractory disease having failed multiple traditional treatments.

Botulinum toxin has been used for the treatment of many medical conditions, most commonly that of muscle spasticity. Its effectiveness stems from its ability to inhibit acetylcholine release at the neuromuscular junction through cleavage of SNARE (soluble $n$-ethylmaleimide sensitive fusion attachment receptor) proteins, thus decreasing muscle contraction [11]. The effects last until the body repairs the effected neuromuscular junctions, a process which lasts about three months [12]. When appropriately dosed and administered, 
adverse effects are usually self-limited and due to mild muscle weakness or injection site reactions.

While botulinum toxin injection is a wellestablished method for the treatment of muscular spasticity, we believe this is the first described use of its treatment in Dupuytren's contracture. In this case, the patient reported improvement in both hand function and pain. The question here is how botulinum toxin, which works on the neuromuscular junction, could improve symptoms of a fibroproliferative disease of the fascia. We believe that the results seen in this case are not the result of a decrease in fascial contracture, but rather through the toxin's effect on the intrinsic hand muscles, where it is already a proven treatment choice. By relaxing these muscles, the patient had an overall improvement of her hand range of motion and function. Also of importance, is the significant relief of pain that the patient experienced. There has been a growing interest in the use of botulinum toxin for the treatment of chronic and neuropathic pain conditions due to inhibitory effects on pain signals substance $P$, CGRP (calcitonin gene-related peptide), glutamate and TRPV1 (transient receptor potential cation channel subfamily $\mathrm{V}$ member 1 ). This is likely the mechanism for which our patient experienced pain-relief.

\section{References}

1. Shum DT, McFarlane RM. Histogenesis of Dupuytren's disease: An immunohistochemical study of 30 cases. J Hand Surg Am 1988; $13 \mathrm{~A}(1): 61-67$. doi:10.1016/0363-5023(88)90202-X.

2. Tan $K$, Withers $A H J$, Tan ST, Itinteag $T$. The role of stem cells in dupuytren's disease: A review. Plast Reconstr Surg - Glob Open 2018;6:1-5. doi:10.1097/GOX.0000000000001777.

3. Townley WA, Baker R, Sheppard N, et al. Dupuytren's contracture unfolded. $\mathrm{Br}$ Med J 2006;332:397-400. doi:10.1136/bmj.332.7538.397

4. Tubiana R. Dupuytren's disease of the radial side of the hand. Hand Clin 1999; 15(1):149159.

5. Larocerie-Salgado J, Davidson J. Nonoperative treatment of PIPJ flexion contractures

\section{Conclusions}

Multiple treatment options have been investigated for Dupuytren's contracture. Many of the conservative approaches have proven to be ineffective, while more invasive techniques still often fail to provide definitive results. For this reason, there is a need for additional research into new ways of managing the condition. We propose the use of the botulinum toxin A injection, an existing and well-tolerated therapy, as a treatment to be considered in Dupuytren's myofascial contracture. Its potential to improve overall hand function through relaxation of the intrinsic hand muscles and inhibit pain pathways makes it an appealing option in painful cases that have failed traditional modalities. Further investigation into its effectiveness and tolerance are warranted.

\section{Consent}

Written informed consent was obtained from the patient for publication of this case report. A copy of the written consent is available for review by the Editor-in-Chief of this journal.

\section{Competing interests}

The author(s) declare that they have no competing interests. associated with Dupuytren's disease. J Hand Surg Eur Vol 2012;37:722-727. doi:10.1177/1753193411422680.

6. Ketchum LD, Donahue TK. The injection of nodules of Dupuytren's disease with triamcinolone acetonide. J Hand Surg Am 2000; 25(6):1157-1162. doi:10.1053/jhsu.2000.18493.

7. Ball C, Izadi D, Verjee LS, Chan J, Nanchahal J. Systematic review of non-surgical treatments for early dupuytren's disease. BMC Musculoskelet Disord 2016; 17(1):345. doi:10.1186/s12891-016-1200-y.

8. Feldman G, Rozen N, Rubin G. Dupuytren's contracture: Current treatment methods. Isr Med Assoc J 2017;19:648-650.

9. Peimer CA, Blazar $P$, Coleman $S$, et al. Dupuytren Contracture Recurrence Following Treatment With Collagenase Clostridium 
Histolyticum (CORDLESS [Collagenase Option for Reduction of Dupuytren Long-Term Evaluation of Safety Study]): 5-Year Data. J Hand Surg Am 2015; 40(8):1597-1605. doi:10.1016/j.jhsa.2015.04.036.

10. Rayan GM. Nonoperative treatment of Dupuytren's disease. J Hand Surg Am 2008; 33(7):1208-1210. doi:10.1016/j.jhsa.2008.05.027.

11. Brown EA, Schütz SG, Simpson DM. Botulinum toxin for neuropathic pain and spasticity: an overview. Pain Manag 2014; 4(2):129-151. doi:10.2217/pmt.13.75.

12. De Paiva A, Meunier FA, Molgó J, Aoki KR, Dolly JO. Functional repair of motor endplates after botulinum neurotoxin type A poisoning: Biphasic switch of synaptic activity between nerve sprouts and their parent terminals. Proc Natl Acad Sci U S A 1999; 96(6):3200-3205. doi:10.1073/pnas.96.6.3200. 